\title{
Circular RNA circ-4099 is induced by TNF-a and regulates ECM synthesis by blocking miR-616-5p inhibition of Sox9 in intervertebral disc degeneration
}

\author{
Hua Wang ${ }^{1}$, Peiheng He${ }^{1}$, Hehai Pan ${ }^{1}$, Jun long ${ }^{1}$, Jianru Wang ${ }^{1}$, Zemin Li ${ }^{1}$, Hui Liu' ${ }^{1}$ Weiying Jiang ${ }^{2}$ and
} Zhaomin Zheng ${ }^{1}$

\begin{abstract}
Circular RNAs (circRNAs) play important roles in the initiation and development of different diseases. Here, we detected their role in intervertebral disc (IVD) degeneration. An Arraystar human circular RNA microarray assay was used to detect circRNAs in normal and degenerated human IVD nucleus pulposus (NP) tissues. The role of circ-4099 in IVDD and its mechanism were evaluated by qRT-PCR and gain-of-function/loss-of-function studies. Interaction networks for competing endogenous RNAs (ceRNAs), miRNAs, and miRNA target gene were detected by bioinformatics analysis, RNA

immunoprecipitation and luciferase assay. Expression of seventy-two circRNAs were increased by more than twofold in degenerated NP tissues. qRT-PCR showed that the expression of circ-4099 in NP tissues was consistent with that of the array screening. Over-expression of circ-4099 increased the expression of Collagen II and Aggrecan and decreased the secretion of the pro-inflammatory factors IL-1 $\beta$, TNF- $a$, and PGE2. TNF- $a$ treatment increased circ-4099 expression in NP cells. NF-KB/MAPK inhibitors or shRNAs abolished the inductive effects of TNF-a on circ-4099 expression. We further demonstrated that circ-4099 was able to function as a "sponge" by competitively binding miR-616-5p, which reversed the suppression of Sox9 by miR-616-5p. We used DNA pull-down and spectrometry experiments to show that TNF-a can promote circ-4099 transcription through upregulation of GRP78. We provide the first evidence that shows circRNAs are differentially expressed in degenerated and normal NP tissues. Circ-4099 may play a role in a protective mechanism and be part of a compensatory response that maintains the synthesis and secretion of the extracellular matrix in NP cells and might be a protective factor in IVD degeneration as well as restore NP cell function.
\end{abstract}

\section{Introduction}

Low back pain (LBP) is one of the most common and costly health problems, affecting $\sim 80 \%$ of the population during their life time, with total costs exceeding $\$ 100$

\footnotetext{
Correspondence: Hua Wang (wangxucheng@gmail.com) or Weiying. Jiang (Jiangwy@mail.sysu.edu.cn) or Zhaomin. Zheng (zhengzm1@163.com) 'Department of Orthopedics, First Affiliated Hospital of Sun Yat-sen University, 58 Zhongshan Second Road, Guangzhou, China

${ }^{2}$ Department of Medical Genetics, Zhongshan School of Medicine and Center for Genome Research, Sun Yat-Sen University, 74 Zhongshan Second Road, Guangzhou, China

These authors contributed equally: Hua Wang and Peiheng He.
}

billion per year ${ }^{1,2}$. Intervertebral disc (IVD) degenerationrelated disorder is the major contributor of LBP and associated disabilities ${ }^{3}$. The hallmark of IVD degeneration is decreased cell density ${ }^{4}$ and extracellular matrix (ECM $)^{5}$. Nucleus pulposus (NP) cells that produce proteoglycan aggrecan and collagens to form complex ECM play a key role in maintaining disc health ${ }^{6}$. IVD degeneration results in increased production of degradative enzymes ${ }^{2}$ together with decreased synthesis of $\mathrm{ECM}^{7}$. These catabolic processes are thought to be mediated by a number of cytokines, including TNF- $\alpha$ and IL- $1 \beta^{8}$. Thus, TNF- $\alpha$ and

\section{(c) The Author(s) 2018}

\footnotetext{
Open Access This article is licensed under a Creative Commons Attribution-NonCommercial-NoDerivatives 4.0 International License, which permits any non-commercial CC. Creative Commons license. You do not have permission under this license to share adapted material derived from this article or parts of it. The images or other third party material in this article are included in the article's Creative Commons license, unless indicated otherwise in a credit line to the material. If material is not included in the article's Creative Commons license and your intended use is not permitted by statutory regulation or exceeds the permitted use, you will need to obtain permission directly from the copyright holder. To view a copy of this license, http://creativecommons.org/licenses/by-nc-nd/4.0/.
} 
IL-1 $\beta$ are the most selected reagents to induce IVD degeneration cellular models in vitro ${ }^{8}$. Although many factors have been demonstrated to participate in the IVD degeneration process, the exact pathophysiology regarding IVD degeneration requires further investigation.

Circular RNAs (circRNAs), which were discovered more than 20 years ago, are a novel type of widely expressed noncoding RNA (ncRNA) that are different from linear RNAs (miRNAs are also linear) and represent an understudied class of ncRNAs ${ }^{9}$. circRNAs form a covalently closed continuous loop, are conserved across species, and usually show tissue-specific or developmental stage-specific expression ${ }^{10}$. circRNAs are more stable than linear RNAs because of their higher nuclease stability, which gives them great advantages as novel biomarkers in clinical applications ${ }^{11}$. circRNAs have been shown to function as natural miRNA sponge transcripts that compete for binding with endogenous RNAs in diverse species ${ }^{12}$. The interactions between circRNAs and miRNAs suggest the potential importance of circRNAs in disease regulation. Hansen et $\mathrm{al}^{13}$. showed a highly expressed circRNA in human and mouse brain tissue and the degeneration process. Li et $\mathrm{al}^{11}$. demonstrated that cir-ITCH expression was low in esophageal cancer compared to peri-tumoral tissue, which indicated that cir-ITCH may have an inhibitory effect on esophageal cancer. Hansen et $\mathrm{al}^{12}$. demonstrated that ciRS7 probably serves as a crucial factor that is significantly engaged in neuronal function and is responsible for neurological disorders and brain tumor development. Ghosal et $\mathrm{al}^{14}$. revealed circRNA SNPs related to a wide range of diseases, including esophageal, gastric, and prostate cancer, multiple sclerosis, and neurodegenerative diseases, such as Parkinson's disease, Alzheimer's disease, among others. However, the roles of circRNAs in IVD degeneration remain a largely uncharted territory.

In this study, we performed circRNA expression profiling analysis in degenerated compared with nondegenerated human IVD NP tissues using an Arraystar human circRNA microarray. This array is the first commercially available circRNA microarray (Arraystar, Inc., USA) designed for the global profiling of human circRNAs. Our data also rely on published paired-end sequencing data and splice sites $^{15,16}$.

\section{Materials and Methods}

\section{Collection and grading of human NP Tissues}

Degenerated IVD tissue samples were collected as surgical waste from patients undergoing spinal surgery in our hospital. Control non-degenerated samples were obtained from patients suffering from vertebral fracture or scoliosis (Supplemental Table 1). Samples were collected according to the approval of the local ethics committee, and informed consent was obtained from every patient or family members before collection. Assessment of IVD degeneration was performed according to the description by Pfirrmann et $\mathrm{al}^{17}$. Samples were divided into two groups, six non-degenerated (grade I/II) and six degenerated (grade IV/V) samples.

\section{Isolation of NP cells and treatment}

Human NP cells were isolated from non-degenerated IVD tissues from spine fracture patients undergoing spinal surgery using a previously described method ${ }^{18}$. NP cells were maintained in Dulbecco's Modified Eagle Medium (DMEM) and 10\% fetal bovine serum (FBS) supplemented with antibiotics. To investigate the effect of cytokines, cells were treated with TNF- $\alpha(50 \mathrm{ng} / \mathrm{ml}$, Peprotech, NJ) for $24 \mathrm{~h}$.

\section{Array data production}

Total RNA from NP tissues was extracted using the Trizol reagent (Invitrogen) and then purified using a NucleoSpin ${ }^{\circledR}$ RNA clean-up Kit (Macherey-Nagel). Extracted total RNA was quantified with a NanoDrop ND-1000 spectrophotometer (Thermo Scientific). The sample preparation and microarray hybridization were performed by KangChen, Inc., based on Arraystar's standard protocols. Briefly, total RNA was digested with Rnase R (Epicentre, Inc.) to remove linear RNAs and enrich for circRNAs. Then, the enriched circRNAs were amplified and transcribed into fluorescent cRNA utilizing a random priming method (Arraystar Super RNA Labeling Kit; Arraystar). The labeled cRNAs were hybridized onto the Arraystar Human circRNA Array $(8 \times 15 \mathrm{~K}$, Arraystar $)$. After washing the slides, the arrays were scanned by the Agilent Scanner G2505C.

\section{Differential expression analysis}

The Agilent Feature Extraction software (version 11.0.1.1) was used to analyze the acquired array images. Quantile normalization and subsequent data processing were performed. Differentially expressed circRNAs with statistical significance between the two groups were identified through Volcano Plot filtering. Differentially expressed circRNAs between two samples were identified through fold change filtering. Hierarchical Clustering was performed to show the distinguishable circRNA expression patterns among samples.

\section{miRNA prediction and co-expression networks}

circRNA/miRNA interactions were predicted with Arraystar's homemade miRNA target prediction software (Rockville, USA), whose principles are based on the TargetScan and miRanda prediction algorithms. The differentially expressed circRNAs within the comparisons were annotated in detail with the circRNA/miRNA interaction information. To further elucidate correlations between the circRNAs and miRNAs, potential 
circRNA-miRNA-mRNA interaction analysis was conducted by Cytoscape. The size of each node represents the number of putative miRNAs functionally connected to each circRNA.

\section{Plasmid construction}

We constructed the circRNA over-expression plasmid used in this study. The construct method was previously published $^{12}$. The resulting construct (pcDNA3.1-cirDENN5A) was verified by direct sequencing with the cirDENN5A -F (CGG AAT TCT GAA ATA TGC TAT CTT AC AGC ATT ATG CCA GTA CATA CAG GCT) and cir-DENN5A -R (CGG GAT CCT CAA GAA AAA ATA TAT TCA CGC TGT GAG TAG AGC AGG ATT TGA) primers.

The full-length sequences of DENN5A and the Sox9 gene were amplified using PCR methods. The reporter promoter contains cir-DENN5A, Sox9-wild type and Sox9-mutation and was constructed with a previous method; the resulting construct was verified by direct sequencing with the following primers: (1) cir-DENN5A -F (AAA CTC GAG CAT TAT GCC AGT ACA TAC AGG CTT CTAA) and cir-DENN5A -R (AAA GCG GCC GCG CTG TGA GTA GAG CAG GAT TTG AA), (2) Sox9-WT -F (AAA ACT CGA GGG AGG CCT CCC ACG AAG) and Sox9-WT -R (AAA AGC GGC CGC TTT CTT TTT AAT GCA ATG TAT ATT TAT TGT AAA CA), and (3) Sox9-MT -F (CCA TTA CCA AAA ACT CGG GAT TTA TAC ATA TTT TTA GAT AAA ATT AAA TGC TC) and Sox9-MT -R (ATC CCG AGT TTT TGG TAA TGA ATC ATA CAC AGT ACA TAC TAA AAA TAT T).

\section{Site-directed mutagenesis}

The Sox9-W reporter plasmid was used to mutate the miR-616-5p site (TTTTGAG to AAAACTC). Mutants were generated with the QuickChange II XL site-directed mutagenesis kit (Stratagene, Wilmington, DE, USA) using forward and reverse primer pairs containing the desired mutation following the manufacturer's instructions. The mutations were verified by sequencing.

\section{RNA isolation and qRT-PCR}

Following treatment, total RNA was extracted from NP tissues or NP cells using the Trizol reagent (Invitrogen) as described above. Two micrograms of total DNA-free RNA was used to synthesize cDNA with the SuperScript III cDNA synthesis kit (Invitrogen). Reactions were set up in triplicate in 96-well plates using $1 \mu \mathrm{l} \mathrm{cDNA}$ with PrimeScript $^{\text {TM }}$ RT-PCR Kit (Takara) to which gene-specific forward and reverse PCR primers were added. Each set of samples included a template-free control.

Real-time quantitative reverse transcription-polymerase chain reaction (qRT-PCR) was completed using the Taq
qPCR Master Mix (Promega) on an Mx3005P real-time PCR System (Stratagene, La Jolla, CA) following the manufacturer's instructions. Divergent rather than more commonly used convergent circ-4099primers were designed and synthesized by Sangon Biotech. $\beta$-actin was used to normalize mRNA expression. Melting curves were analyzed to verify the specificity. The following genespecific primer pairs were used: (1) Human- $\beta$-actin, F 5'-GCA TGG GTC AGA AGG ATT CCT-3' and R 5'-TCG TCC CAG TTG GTG ACG AT-3'; (2) Homo Circ-4099, F3 5'-CCA GAG ACC AAG TAC CAA TGAG-3' and R $5^{\prime}$-GTA CTG GCA TAA TGG CTG TGA-3'; (3) p65, F 5'-ACA ACA ACC CCT TCC AAG AAGA-3' and R $5^{\prime}$-AGA GTT TCG GTT CAC TCG G C-3'; (4) IKK $\beta$, F 5'-ATC CAG ATC ATG AGA AGG CTG AC-3' and R 5'-AGC AAG GTG AGG ATG GCA C-3'; (5) COLL2, F 5'-GCC AAA GGT GAA CAA GGA GA-3' and R 5'-TCG AGC ACC TTT AGG ACC AT-3'; (6) Aggrecan, F 5'-GTC GTG GTA AAA GGC ATC G T-3' and R 5'-TGC CTC TTG GAA GGT GAA CT-3'; (7) MMP-3, F 5'-TTC TCG AAT CAC GGA GGA AGC CAA-3' and R5'-ACG ACG CCT TCC ATG GAT CTT CTT-3'; (8) ADAMTS5, F 5'-GTC CAA ATG CAC TTC AGC CAC GAT-3' and R 5'-AAT GTC AAG TTG CAC TGC TGG GTG-3'; and (9) DENND5A (linear + circRNA), -F $5^{\prime}$-TCT ACC ACA TGC ACA ATG CT $-3^{\prime}$ and -R 5'-GTC CCG GCT AAT GTC ATA GG -3'.

\section{Protein extraction and western blotting}

NP cells were placed on ice immediately after treatment, washed with ice-cold PBS, and harvested in mammalian protein extraction reagent buffer (Pierce), as reported earlier by Risbud et $\mathrm{al}^{19}$. Protein concentrations were measured using a BCA Protein Assay Kit (Pierce, USA). Total cellular proteins were resolved on $8-12 \%$ SDSpolyacrylamide gels and transferred via electroblotting to PVDF membranes (Millipore). The membranes were blocked in 5\% non-fat dry milk with TBST and incubated overnight at $4{ }^{\circ} \mathrm{C}$ with antibodies against Aggrecan (ACAN), Collagen II, Sox9, p65, IKK $\beta$, GRP94, and GRP78 from Abcam. After washing with TBST, the membranes were incubated with anti-rabbit or anti-Rat HRP-conjugated secondary antibodies and detected with ECL Plus (Millipore, USA) reagent. The results were then quantified with a multigauge densitometry system (Fujifilm, Tokyo, Japan).

\section{Transient transfections and luciferase assays}

NP cells were transfected with the reporter plasmids described above using Lipofectamine 2000. Cells were cotransfected with miRNAs according to the manufacturer's instructions. Each group included three replicates and triplicate independent experiments were performed. In the luciferase screening assay, we used one internal 
Table 1 Selected patient characteristics

\begin{tabular}{|c|c|c|c|c|c|}
\hline & Gender & Age & Diagnosis & Operation level & IVDD classification \\
\hline 1 & M & 30 & L3 burst fracture & L2-4 & । \\
\hline 2 & $\mathrm{~F}$ & 32 & L2 burst fracture & L1-3 & $\|$ \\
\hline 3 & M & 35 & L2 burst fracture & L1-3 & I \\
\hline 4 & M & 38 & L2 burst fracture & L1-3 & $\|$ \\
\hline 5 & $\mathrm{~F}$ & 28 & Scoliosis & $\llcorner 4-5$ & $\|$ \\
\hline 6 & $\mathrm{~F}$ & 26 & L3 burst fracture & $\mathrm{L} 2-4$ & । \\
\hline 7 & M & 48 & Intervertebral disc herniation (L5/S1) & L5-S1 & IV \\
\hline 8 & M & 52 & Intervertebral disc herniation ( $\mathrm{L} 5 / \mathrm{S} 1)$ & L5-S1 & IV \\
\hline 9 & M & 50 & Lumbar spondylolisthesis (L5) & L5-S1 & V \\
\hline 10 & $\mathrm{~F}$ & 49 & Lumbar spondylolisthesis (L5) & L5-S1 & V \\
\hline 11 & $\mathrm{~F}$ & 51 & Lumbar spondylolisthesis (L4) & $\llcorner 4-5$ & V \\
\hline 12 & M & 48 & Lumbar spondylolisthesis (L5) & L5-S1 & V \\
\hline
\end{tabular}

control (RL reporter) and two negative controls (miRNA control and luciferase reporter lacking the circDENN5A 3'-UTR). Each miRNA or NC-RNA was co-transfected with RL reporter and FL reporter with or without the cirDENN5A 3'-UTR. For comparison, the FL activity was first normalized with RL activity. The effect of each miRNA on luciferase reporter with the circDENN5A 3'UTR was then normalized with that of luciferase reporter without the circDENN5A 3'-UTR. Finally, the fold change was calculated for each miRNA compared with NC.

\section{Lentiviral particle production and viral transduction}

HEK 293T cells were seeded into $10 \mathrm{~cm}$ plates in DMEM with 10\% FBS 2 days before transfection. Cells were transfected with $2.5 \mu \mathrm{g}$ of sh-control, sh-p65, or shIKK $\beta$ plasmids along with $1.875 \mu \mathrm{g}$ psPAX2 and $0.625 \mu \mathrm{g}$ pMD2.G. After $16 \mathrm{~h}$, the transfection media was removed and replaced by DMEM with 5\% FBS and penicillin/ streptomycin. Lentiviral particles were harvested at $48 \mathrm{~h}$ post-transfection. NP cells were plated in DMEM with 5\% FBS. Cells in $10 \mathrm{~cm}$ plates were transduced with $5 \mathrm{ml}$ of viral particle-conditioned media with $6 \mu \mathrm{g} / \mathrm{ml}$ polybrene. After $24 \mathrm{~h}$, the conditioned media was replaced by DMEM with $5 \%$ FBS. Cells were collected for RNA or protein extraction after treatment.

\section{RNA FISH}

hNP cells cultured on a glass coverslip were fixed in $4 \%$ paraformaldehyde for $30 \mathrm{~min}$ and permeabilized with 0.1 $\%$ Triton X-100 for $30 \mathrm{~min}$. The cells were washed three times with PBS and treated with pre-hybridization buffer ( $2 \times$ saline-sodium citrate) containing $10 \%$ formamide. Hybridization was performed using a similar buffer, but with the addition of competitor RNA (tRNA) and competitor protein (BSA) to reduce background. The FITC-labeled circ-4099 and miR-616-5p probe.

\section{RNA-binding protein immunoprecipitation assay}

RNA-binding protein immunoprecipitation (RIP) experiments were performed using the Magna RIP RNABinding Protein Immunoprecipitation Kit (Millipore, Bedford, MA). The Ago-RIP assay was conducted in hNP cells stably expressing Flag-AGO2 or Flag-GFP. We first constructed the lentivirus vectors harboring Flag-Ago2 or FlagGFP and established two stable cell lines after lentivirus transduction in hNP cells (denoted as Flag-GFP and FlagAgo 2 cells). Approximately $10^{7}$ cells were pelleted and resuspended in an equal pellet volume of RIP Lysis Buffer $(\sim 100 \mathrm{ml})$ plus protease and RNase inhibitors. The cell lysates $(100 \mathrm{ml})$ were incubated with $5 \mathrm{mg}$ of control mouse IgG-coated or antibody against Flag peptide-coated (Sigma-Aldrich) beads with rotation at $4{ }^{\circ} \mathrm{C}$ overnight. After treating with proteinase $\mathrm{K}$, the immunoprecipitated RNAs were extracted with the RNeasy MinElute Cleanup Kit (Qiagen) and reverse transcribed using the Prime-Script RT Master Mix (TaKaRa). The abundance of miR-616-5p and Circ-4099 was detected by qRT-PCR assay.

\section{Biotin-coupled miRNA capture}

The biotin-coupled miRNA pull-down assay was performed as described previously. Briefly, 3'-end biotinylated miR-616 mimic or control RNA (RiboBio) were transfected into hNP cells at a final concentration of 20 $\mathrm{nM}$ for 1 day. The biotin-coupled RNA complexes were pulled down by incubating the cell lysates with streptavidin-coated magnetic beads (Life Technologies). The abundance of circDENN5A in the bound fractions was evaluated by qRT-PCR analysis. 
Table 2 Top 10 differentially expressed circRNAs (degenerated vs. non-degenerated)

\begin{tabular}{|c|c|c|c|c|c|c|}
\hline circRNA & Regulation & Type & FC (abs) & Alias & FDR & $P$-value \\
\hline hsa_circRNA_100759 & Up & Exonic & 6.0436796 & circ_0004099 & 0.001985008 & 2.80285E-05 \\
\hline hsa_circRNA_103555 & Up & Exonic & 5.5301653 & circ_0068610 & 0.083401104 & 0.025051229 \\
\hline hsa_circRNA_102113 & Up & Exonic & 5.3087065 & circ_0002069 & 0.023900268 & 0.002867487 \\
\hline hsa_circRNA_101353 & Up & Exonic & 4.5647371 & circ_0031897 & 0.070983991 & 0.018858289 \\
\hline hsa_circRNA_104574 & Up & Exonic & 4.0113787 & circ_0083756 & 0.070942531 & 0.018794621 \\
\hline hsa_circRNA_103029 & Up & Exonic & 3.8610047 & circ_0059955 & 0.016096515 & 0.001457627 \\
\hline hsa_circRNA_100719 & Up & Exonic & 3.6146979 & circ_0020390 & 0.015623803 & 0.001380433 \\
\hline hsa_circRNA_104135 & Up & Exonic & 3.5263885 & circ_0007874 & 0.098387237 & 0.032936772 \\
\hline hsa_circRNA_400101 & Up & Intronic & 3.5095173 & circ_0092328 & 0.053147953 & 0.011768583 \\
\hline hsa_circRNA_103541 & Up & Exonic & 3.5020867 & circ_0068462 & 0.046516597 & 0.009053098 \\
\hline hsa_circRNA_103225 & Down & Exonic & 3.105779 & circ_0063331 & 0.045605649 & 0.00875982 \\
\hline hsa_circRNA_104194 & Down & Exonic & 2.8688053 & circ_0004712 & 0.028477579 & 0.00389463 \\
\hline hsa_circRNA_101004 & Down & Exonic & 2.6683336 & circ_0000375 & 0.112304139 & 0.040901878 \\
\hline hsa_circRNA_000596 & Down & Antisense & 2.6262258 & circ_0000661 & 0.047669869 & 0.009363936 \\
\hline hsa_circRNA_101412 & Down & Exonic & 2.5754826 & circ_0005252 & 0.041823488 & 0.007759216 \\
\hline hsa_circRNA_104040 & Down & Exonic & 2.54998 & circ_0075410 & 0.098387237 & 0.032945284 \\
\hline hsa_circRNA_400027 & Down & Intronic & 2.5112 & circ_0092367 & 0.06739006 & 0.017092378 \\
\hline hsa_circRNA_103818 & Down & Exonic & 2.4968942 & circ_0072279 & 0.107230858 & 0.037801246 \\
\hline hsa_circRNA_103639 & Down & Exonic & 2.3998995 & circ_0007646 & 0.070058343 & 0.018376124 \\
\hline hsa_circRNA_100024 & Down & Exonic & 2.2937773 & circ_0009135 & 0.106912695 & 0.03757954 \\
\hline
\end{tabular}

Biotin-promoter pull-down assay and mass spectrometry

Double-stranded circDENN5A promoter was synthesized by PCR and labeled with biotin-14-dCTP according to the manufacturer's instructions (19518-018, Invitrogen, Grand Island, NY, USA). Biotin-labeled DNA was diluted in $10 \mathrm{mM}$ Tris- $\mathrm{HCl}, 10 \mathrm{mM} \mathrm{MgCl}_{2}, 25 \mathrm{mM} \mathrm{NaCl}$, and 10 $\%$ glycerol and incubated with nuclear protein extract at 4 ${ }^{\circ} \mathrm{C}$ for $12 \mathrm{~h}$. In total, $100 \mu \mathrm{l}$ streptavidin-linked magnetic beads (88816, Thermo Scientific, Waltham, MA USA) were used to pull down the biotinylated DNA at room temperature for $2 \mathrm{~h}$. The bead-DNA-protein complexes were then washed with $1 \times$ binding and washing buffer $(5$ $\mathrm{mM}$ Tris- $\mathrm{HCl}, 1 \mathrm{M} \mathrm{NaCl}, 0.5 \mathrm{mM}$ EDTA, and $0.005 \%$ Tween 20 four times. The proteins were precipitated and diluted in $100 \mu \mathrm{l}$ protein lysis buffer. One-shot mass spectrometry analyses were then performed to analyze the purified nuclear proteins.

\section{Results}

circRNAs are differentially expressed in degenerated and non-degenerated NP tissues

Given the high abundance of circRNAs in other tissues, we aimed to evaluate the role of circRNAs in the IVD degeneration process. We analyzed human circRNA expression in degenerated versus non-degenerated IVD NP tissues(Table 1). Among the 5397 circRNAs, 21 were downregulated and 51 upregulated by more than twofold in degenerated compared with non-degenerated NP tissues $(P<0.05)$ (Table 2) (Fig. 1a, b). Hierarchical clustering analysis indicated distinguishable circRNA expression profiles between the samples and homogeneity within groups (Fig. 1c). Circ-4099, circ-68610, circ-2069, and circ92328 as well as circ-4099 were used for further analysis.

\section{circRNA amplification}

To validate the efficacy and specificity of the divergent primers to detect the four circRNAs, we performed realtime RT-PCR experiments. A single-peak melting curve indicated that there were no primer dimers or nonspecific amplification. The sequence of the amplified circ4099 product was completely consistent with CircBase (Fig. 1d, other sequence data not shown). These results showed that we could reliably detect and quantify circ4099 in NP tissues or cells by qRT-PCR.

\section{The localization of circ-4099 in NP cells}

We next investigated the localization of this circRNA in NP cells. Fluorescence in situ hybridization (FISH) against 


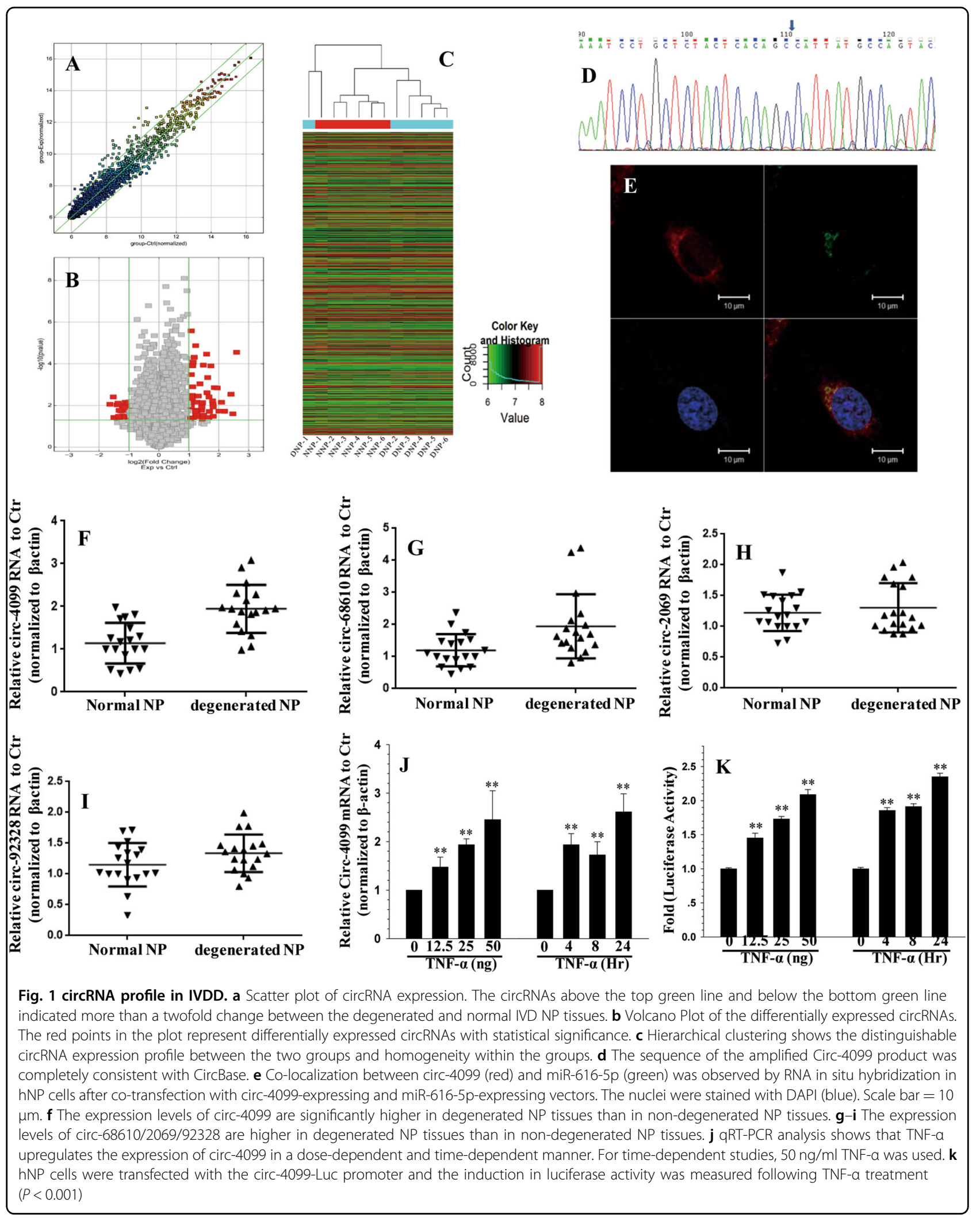




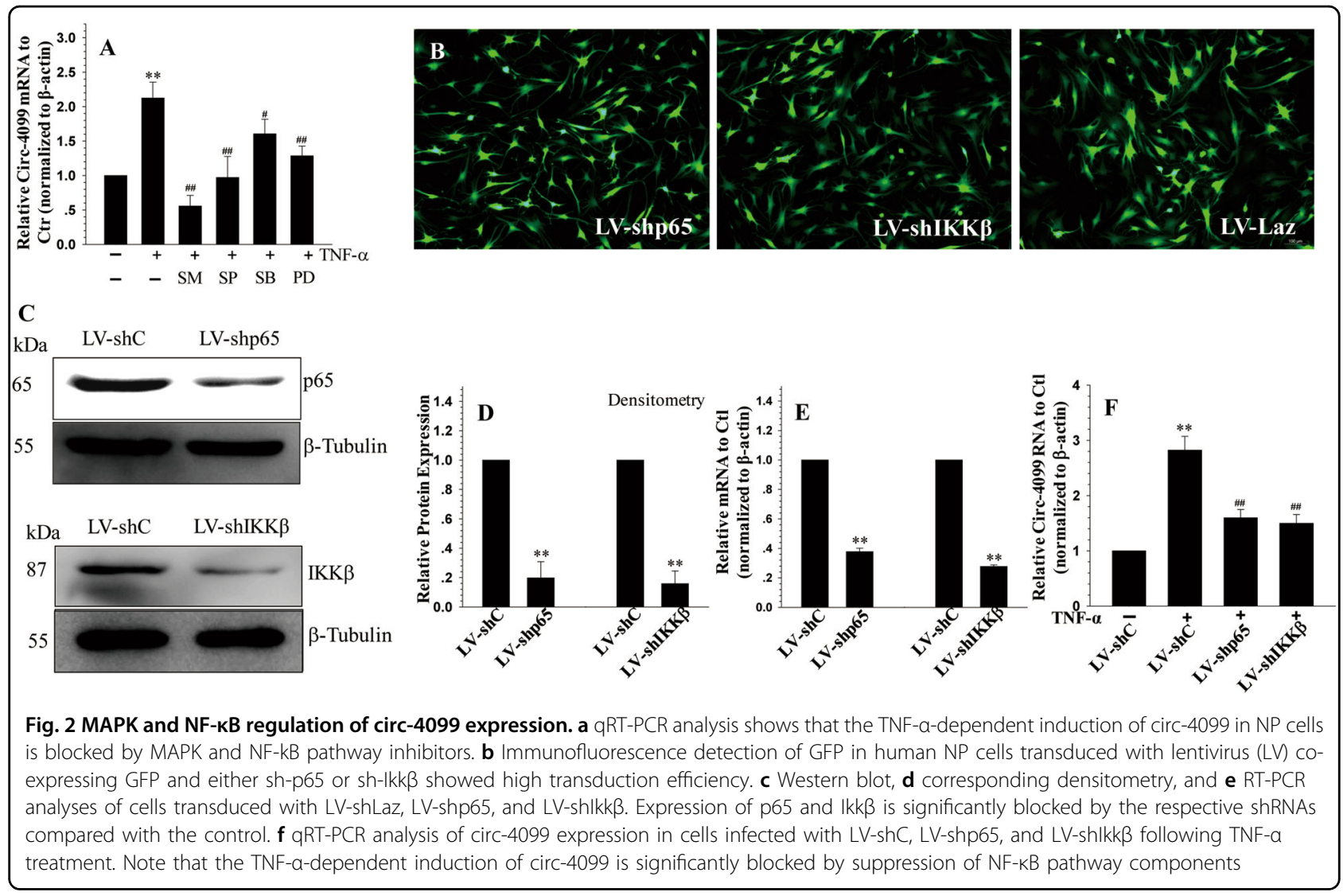

circ-4099 demonstrated that the circular form of DENND5A preferentially localized in the cytoplasm (Fig. 1e). Taken together, our results show that circ-4099 is an abundant and stable circRNA expressed in different human cells.

Increased Circ-4099 expression in degenerated NP tissues

To validate the circRNA microarray results, we selected circ-4099, circ-68610, circ-2069, and circ-92328, which exhibited significant changes in expression among the differentially expressed circRNAs and performed qPCR to analyze the changes in expression. The data confirmed that circ-4099 was upregulated in degenerated NP tissues with 2.5 -fold change by qPCR $(P<0.001$; Fig. 1 f). The expression of circ-68610 (Fig. 1g), circ-2069 (Fig. 1h), and circ-92328 (Fig. 1i) were also increased in degenerated NP tissues, but were not as consistent as circ-4099, so circ4099 was used for further analysis.

TNF- $\alpha$ induced the expression of circ-4099 in the NP cells

The cytokine TNF- $\alpha$ was used to establish a cellular model of IVD degeneration in vitro ${ }^{8}$. To test the premise that cytokines involved with IVD degeneration control circ-4099, NP cells were treated with TNF- $\alpha$ and the expression of circ-4099 was analyzed. qRT-PCR analysis showed that TNF- $\alpha$ upregulated circ-4099 expression in a dose-dependent and time-dependent manner. For timedependent studies, 50 ng/ml TNF- $\alpha$ was used. (Fig. 1j)

To further investigate whether cytokines enhance circ4099 activity in human NP cells, we transfected cells with the circ-4099-Luc wild-type reporter and measured their activity following TNF- $\alpha$ treatment. The results of these experiments showed that TNF- $\alpha$ increased the activity of the circ-4099-Luc reporter in a dose-dependent and timedependent manner. (Fig. 1k)

\section{TNF-a promotes circ-4099 expression through the MAPK and NF-KB pathways}

As the MAPK and NF- $\kappa B$ pathways are the main pathways that induce TNF- $\alpha$, we determined whether the NF- $\kappa B$ and MAPK signaling pathways control the cytokine-dependent induction of circ-4099 expression. NP cells were treated with pathway-specific inhibitors (SM7368 $(10 \mu \mathrm{M})$, SB203580 $(10 \mu \mathrm{M})$, PD98059 $(50 \mu \mathrm{M})$, or SP60025 $(10 \mu \mathrm{M})($ Calbiochem)) $1 \mathrm{~h}$ prior to exposure to TNF- $\alpha$. When induced by TNF- $\alpha$, pretreatment with inhibitors resulted in the suppressed expression of circ4099, with more suppression observed in the NF-kB inhibitor group. (Fig. 2a)

To further elucidate the role of the NF- $\kappa \mathrm{B}$ signaling pathway in the regulation of circ-4099 expression, we silenced the expression of individual NF- $\mathrm{kB}$ signaling 
components and transduced human NP cells with lentivirus co-expressing GFP and p65-shRNA or IKK $\beta$ shRNA. Figure $2 \mathrm{~b}$ shows that there was robust GFP expression in the virally transduced cells, indicating a high level of transduction efficiency and transgenic expression. There was a significant decrease in $\mathrm{p} 65$ and IKK $\beta$ protein (Fig. 2c, d) and mRNA (Fig. 2e) expression in cells transduced with sh-p65 and sh-IKK $\beta$, respectively, when compared to cells transduced with the control shRNA. Importantly, suppression of NF- $\mathrm{kB}$ signaling components significantly decreased the inductive effects of TNF- $\alpha$ on circ-4099 expression (Fig. 2f).

\section{The effects of circ-4099 on ECM degradation and inflammatory cytokine secretion in IVD}

To analyze the effects of circ-4099 on ECM degradation, we examined the effects of circ-4099 over-expression in IVD NP cells. First, we designed a circ-4099 overexpression plasmid with the circ-4099 F (CGG AAT TCT GAA ATA TGC TAT CTT ACA GCA TTA TGC CAG TAC ATA CAG GCT) and circ-4099 R (CGG GAT CCT CAA GAA AAA ATA TAT TCA CGC TGT GAG TAG AGC AGG ATT TGA) primers (Fig. 3a, b). Then, hNP cells were transfected with circ-4099, NC (PLCDH-ciR) or negative control. Total RNA was collected from the transfected cells and qPCR was used to detect the expression of circ-4099 and DENND5A (linear + circRNA). The results showed that circ-4099 over-expression can extremely enhance the expression of circ-4099; the qPCR products were also used to confirm the circ4099 sequences. These data confirmed the validity and efficiency of the circ-4099 over-expression plasmid. (Fig. 3c, d)

Next, we examined whether circ-D over-expression could impact the expression of Collagen II and Aggrecan, two main ECM molecules important for NP tissues. As a consequence of circ-D over-expression, Collagen II, and Aggrecan mRNA expression increased, while MMP3 and ADAMTS5 showed few changes in expression. (Fig. 3e, f)

We further performed western blot to examine the expression of Collagen II and Aggrecan at the protein level. WB and densitometry analysis with three replicates showed that the Collagen II and Aggrecan protein levels were significantly increased by circ- 4099 over-expression and that this effect was reversed by co-transfection with miR-616-5p mimics in NP cells, which indicated that miR-616-5p may function as a circ-4099 sponge. (Fig. 3g, h) These results showed that the increased circ-4099 activities restored functional ECM synthesis in NP cells. However, the regulation of circ-4099 in IVDD and its target gene remain unknown.

We next tested whether circ-4099 can regulate pro-inflammatory genes, including IL- $1 \beta$, TNF- $\alpha$, IL- 6 , and PGE2, in hNP cells. Circ-4099 over-expression significantly suppressed IL- $1 \beta$, TNF- $\alpha$, and PGE2 mRNA levels in hNP cells, but not IL-6 (Fig. 3i, j). Additionally, miR-616-5p mimics can reverse the suppression effects of circ-4099 on pro-inflammatory genes. The release of IL-1 $\beta$, TNF- $\alpha$, IL-6, and PGE2 into medium was detected by ELISA, which showed similar results (Fig. 3k, l). As it has been shown that pro-inflammatory cytokines play a vital role in the pathogenic processes underlying painful IVD degenerative diseases, circ-4099 may regulate disc degeneration and LBP processes.

\section{Annotation of circRNA-miRNA interactions and target genes}

We used gene co-expression networks to predict the circRNA targets. Previous studies have demonstrated that circRNAs were correlated with miRNAs and play a crucial role in fine tuning the levels of miRNA that mediate gene expression regulation by sequestering the miRNAs. To explore how circRNAs might participate in regulating IVD degeneration, we attempted to predict circRNA-microRNA-mRNA interactions using the TargetScan ${ }^{20}$ and miRanda ${ }^{21}$ software. The differentially expressed circRNAs within the comparisons were annotated in detail with the circRNA-miRNA-mRNA interaction information. There were a total of 5 miRNAs and more than 100 mRNAs in the network. In the coexpression networks, each gene corresponds to a node and three genes are connected by a string, indicating a tight correlation between the genes and a potential regulatory relationship. Among the mRNAs, Sox9, which can positively regulate the expression of chondrocyte-specific genes, including aggrecan and collagen $\mathrm{II}^{22}$, was used for further analysis. (S Figs. 1-2).

\section{Circ-4099 serves as a sponge for miR-616-5p}

Given that circRNAs have been shown to act as miRNA sponges and that circ-4099 is abundant and stable in the cytoplasm, we next investigated the ability of circ-4099 to bind to miR-616-5p. We performed RNA-binding protein immunoprecipitation by $\mathrm{qPCR}^{23}$ to determine whether AGO2 was associated with miR-616-5p transcripts, which can bind circ-4099. Lysates from hNP cells were immunoprecipitated with anti-AGO2 antibody $(0.1,1 \mu \mathrm{g})$ or control IgG antibody $(0.1,1 \mu \mathrm{g})$ and subjected to qRTPCR to quantify the expression of miR-616-5p and circ4099. The results showed that the expression of AGO2 protein is high in hNP cells (Fig. 4a, b) and that endogenous miR-616-5p pulled down from Flag-AGO2 cells was specifically enriched by qRT-PCR analysis (Fig. 4c). Circ-4099 was also detected in these RNA-protein complexes (Fig. 4d), which suggested that circ-4099 could bind to miR-616-5p transcripts and be indirectly captured by anti-AGO2. We further performed RNA-RNA pulldown $\mathrm{qPCR}$ tests using a biotin-coupled miR-616-5p 


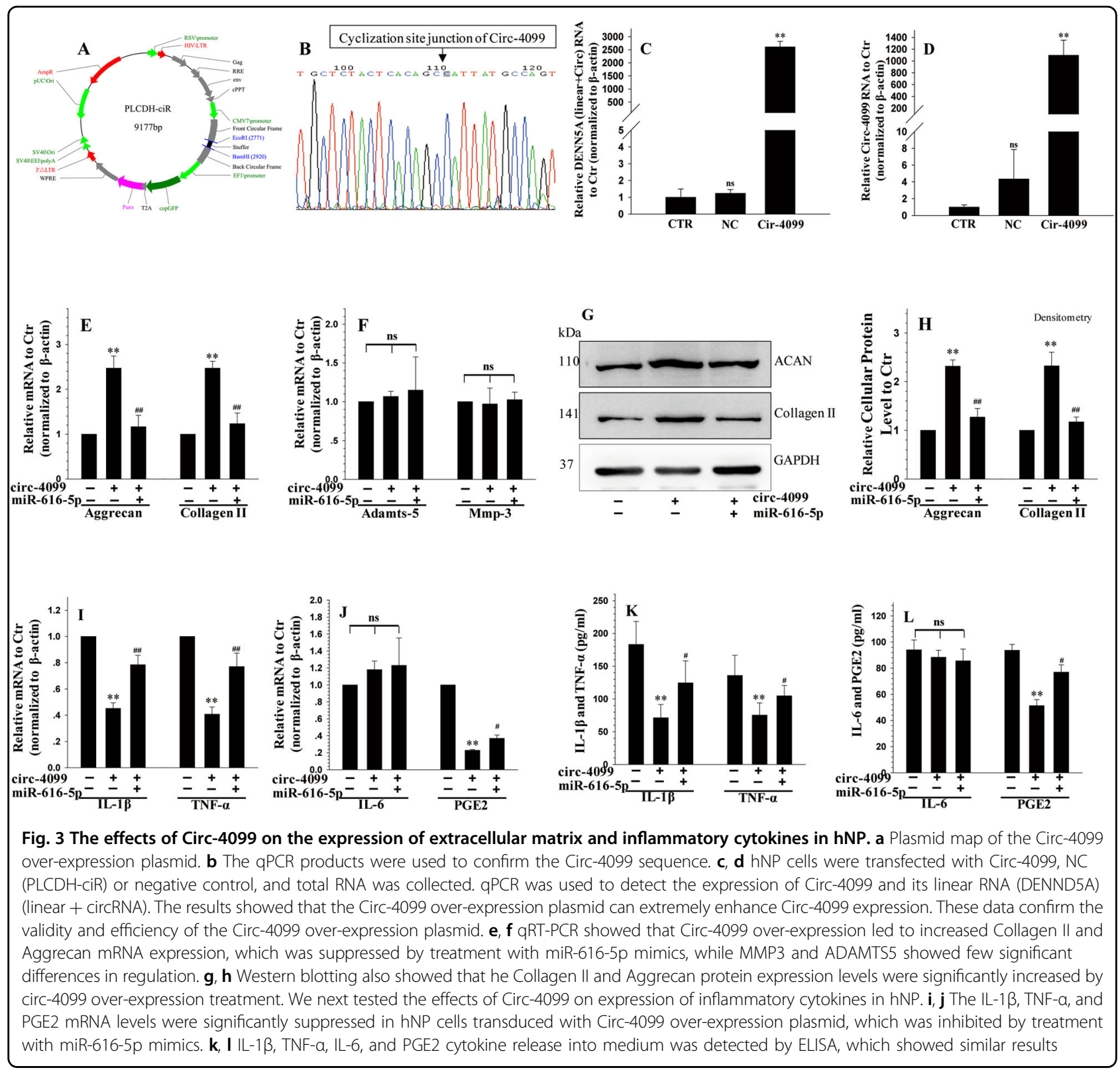

mimic to detect miR-616-5p-related RNAs and proteins. We observed an approximately twofold enrichment of circ-4099 in the miR-616-5p-captured fraction compared with the negative control (Fig. 4e), which demonstrated the binding of circ-4099 to miR-616-5p. WB showed significantly higher AGO2 protein levels in the miR-616$5 \mathrm{p}$-captured fraction compared with the negative control (Fig. 4f). The co-localization experiments (FISH) showed that there is overlapped localization between circ-4099 and miR-616-5p, which supported circ-4099 interacting with miR-616-5p (Fig. 1d). We further constructed a circ4099 fragment and inserted it immediately downstream of the luciferase reporter gene (LUC-circ-4099). We hypothesized that circ-4099-associated miRNAs may potentially inhibit the luciferase activity, presumably via the miRNA-mediated activation of deadenylation and subsequent exonucleolytic degradation. Supporting this hypothesis, we observed that miR-616-5p mimic treatment decreased LUC-circ-4099 plasmid luciferase activity (Fig. 4g). These results suggested that Circ-4099 may serve as a binding platform for AGO2 and miRNAs.

\section{Circ-4099 is targets Sox9 through miR-616-5p as a ceRNA}

It has been reported that circRNAs function as miRNA "sponges" that naturally sequester and competitively suppress miRNA activity ${ }^{12}$. We assumed that circ-4099 functions as a decoy to regulate Sox 9 expression through the same mechanism. According to the network above, 


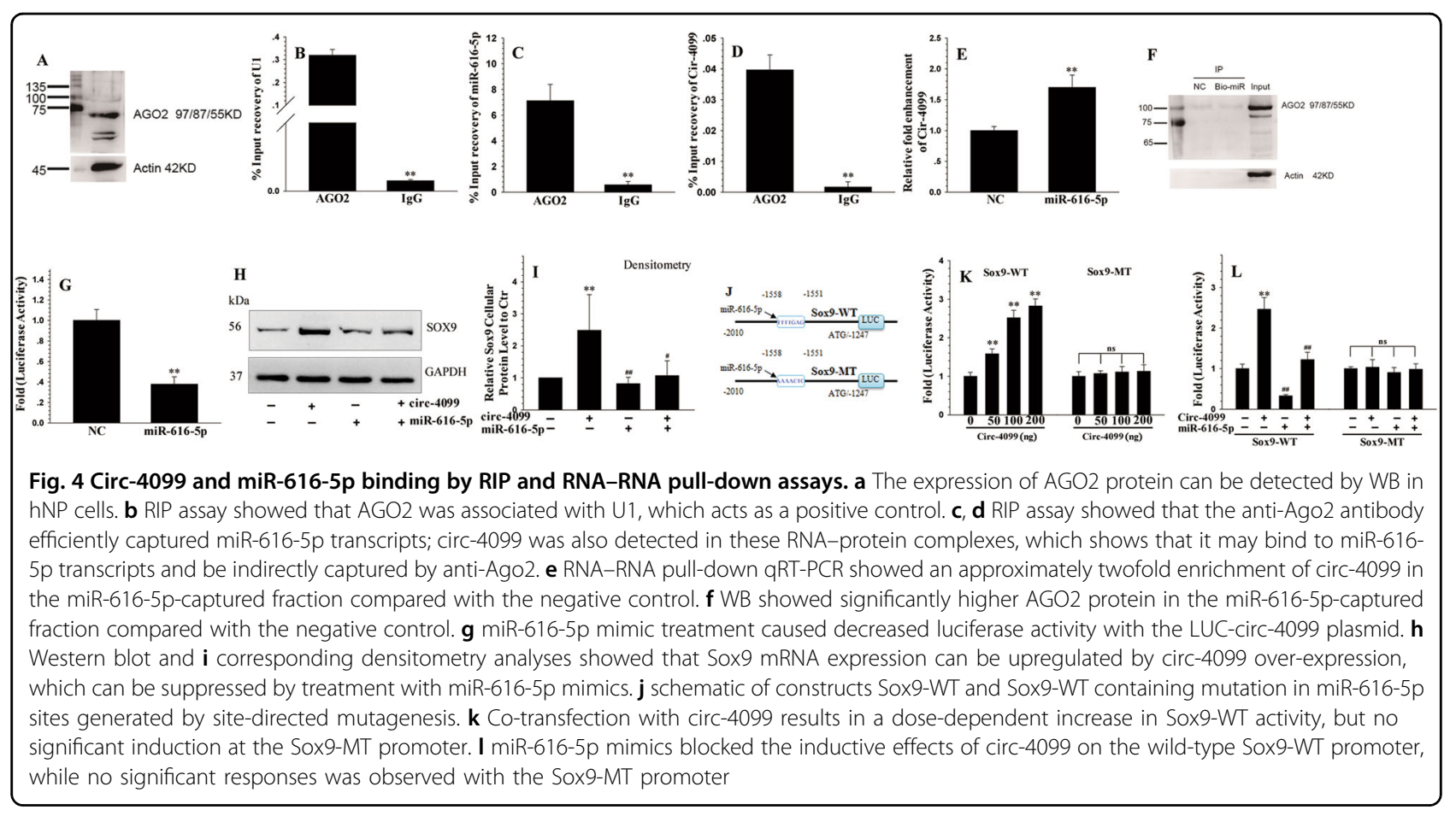

there were five miRNA-binding sites in circ-4099, including miR-508-5p, miR-561-5p, miR-616-5p, miR328-5p, and miR-761 (S Fig. 1). The sequences in the circ-4099 3'-UTR matched these miRNAs. We identified miR-616-5p as circ-4099 and Sox9 targets through informatics analysis. The results showed that miR-616-5p also matched the Sox9 3'-UTR. To analyze the effects of circ-4099 on Sox9 expression, we examined the effects of circ-4099 over-expression in IVD NP cells. qPCR showed that Sox 9 mRNA expression can be upregulated by circ4099 over-expression, which can be suppressed by treatment with miR-616-5p mimics. (Fig. 4h,i) We inferred that circ-4099-induced Sox9 regulation by functioning as a ceRNA (competing endogenous RNAs). Furthermore, a luciferase reporter was constructed to determine whether miR-616-5p can directly target the Sox9 3'-UTR(Fig. 4j). Co-transfection with circ-4099 resulted in a dosedependent increase in Sox9 activity (Fig. 4k), while miR616-5p mimics blocked the inductive effects of circ-4099 on the wild-type Sox 9 promoter (Fig. 4l). To confirm that Sox9 promoter activity is responsive to miR-616-5p, we evaluated the roles of the conserved miR-616-5p-binding sites in controlling Sox9 promoter activity. Using a Sox9-W fragment as a template, we generated a mutant reporter that contained a mutation in the miR-616-5pbinding sites (Fig. 4j) and examined the effects of circ4099 and miR-616-5p mimics on its activity. In contrast to the wild-type promoter, the single site mutants were less responsive to circ-4099 or miR-616-5p mimic treatment (Fig. 4l).
Transcription factors responsible for circ-4099 expression

To investigate the transcription factors that are responsible for circ-4099 expression, we performed a DNA pull-down experiment in nuclear extracts using the biotin-labeled circ-4099 promoter. Nuclear proteins from hNP cells were incubated and pulled down by a biotinlabeled circ-4099 promoter (Fig. 5a). Spectrometry analyses were then performed to analyze the purified nuclear proteins. Among these proteins, we found that glucoseregulated protein had been reported to be related to IVDD (Fig. 5b). Thus, GRP78/94 was used for further analysis. Using WB analysis, we found that GRP78 protein was increased in the nucleus following TNF- $\alpha$ treatment (Fig. 5c, d); in contrast, no NF-kB protein was among the pulled down proteins. WB also showed no bands for p65 or IKK- $\beta$ among the pulled down proteins when the nuclear extracts were incubated with anti-p65 and antiIKK $\beta$ antibodies, respectively (data not shown). This means there may be an indirect contact between the NFКB pathway and circ-4099. After GRP78/94 were individually silenced (Fig. 5e, f), only GRP78 dysregulation obviously affected circ-4099 expression under TNF- $\alpha$ treatment (Fig. 5g).

\section{Discussion}

circRNAs are a new enigmatic class of RNAs with unknown cell-specific functions, and the role of circRNAs in IVD remains largely unknown. To systematically explore the role of circRNAs in human IVD degeneration, we analyzed the differential expression of circRNAs in 


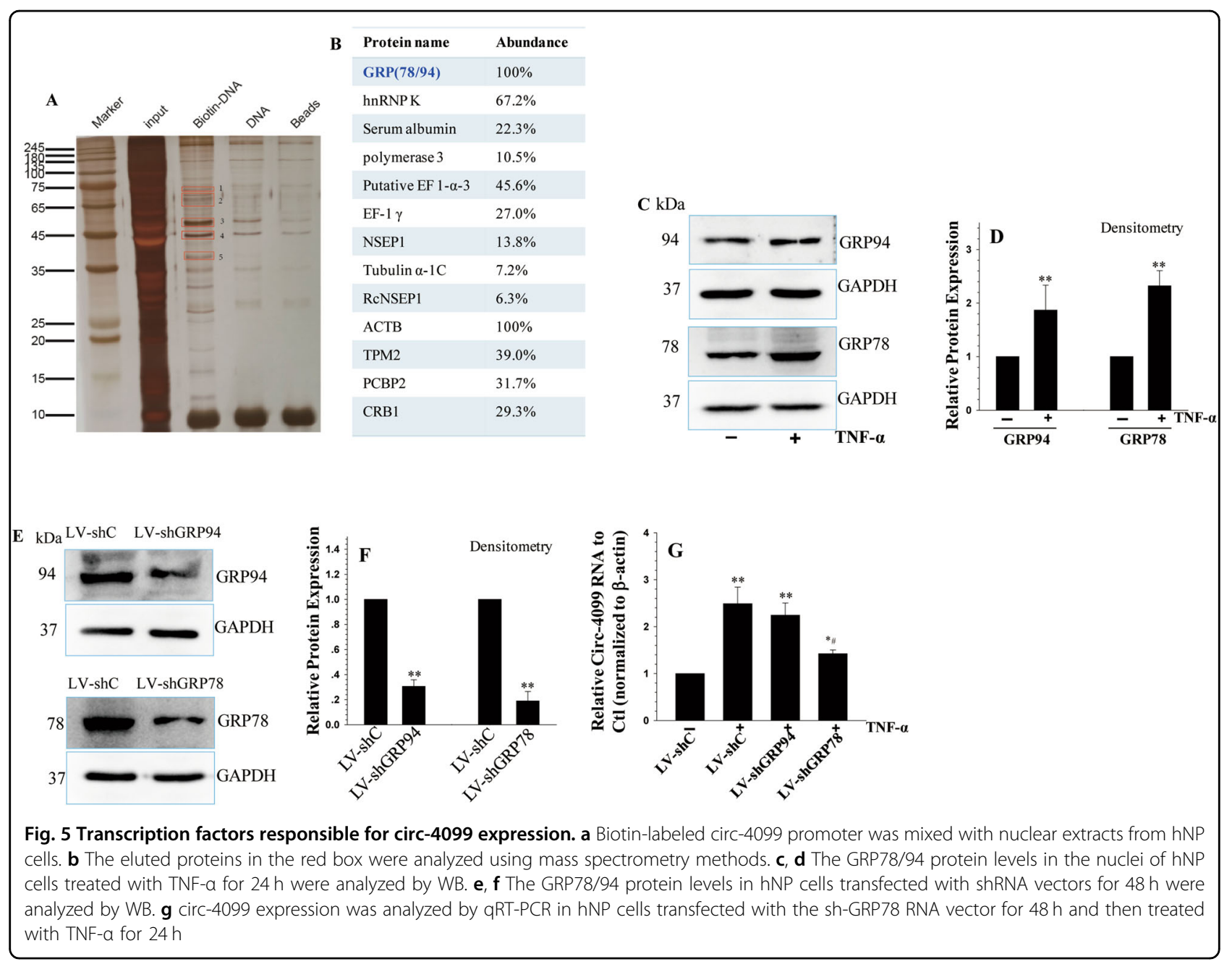

non-degenerated and degenerated IVD NP tissues. We detected thousands of well-expressed stable circRNAs, which often showed tissue-specific or developmental stage-specific expression. Arraystar human circRNA microarray analysis revealed high and specific expression of circRNAs in degenerated IVD NP tissues, with 51 circRNAs upregulated and 21 circRNAs downregulated by more than twofold in degenerated NP tissues, suggesting that circRNAs may play important roles in the initiation and development of IVD degeneration. Through gain-offunction and loss-of-function studies, we found that circ4099 is important in the ECM degradation process in IVD degeneration. A series of experiments illustrated the correlation between circ-4099 and miR-616-5p, demonstrating that circ-4099 plays an important role as an miRNA sponge in the IVDD development process.

Circ-4099 is the most upregulated circRNA among all of the detected circRNAs, displaying a sixfold upregulation in degenerated NP tissues compared with normal NP tissues. Circ-4099 is located on exonic chromosome 11 and is aligned in a sense orientation to the known protein- coding gene DENND5A. DENND5A contains DENN domains and interacts with Rab6, Rab11, and Rab39 as well as small GTPases that are indispensable for intracellular membrane trafficking ${ }^{24}$. DENND5A is a cancer driver candidate gene and functions in epithelial cell polarity as indicated by bioinformatics analysis; it participates in both intracellular trafficking and cell cycle progression $^{25}$. In this study, we found that a circular form of DENN5A is constantly expressed in human NP tissues and was increased expression during IVD degeneration. This suggested that Circ-4099 may play a role in the IVD degeneration process. We designed primers, and head-totail splicing was assayed by real-time RT-PCR after reverse transcription with divergent primers and Sanger sequencing. The predicted circ-4099 head-to-tail junctions were validated to show their high accuracy based on our predictions.

Previous studies have indicated that circRNAs may play important roles in the initiation and development of different diseases, especially aging and degeneration-related diseases. Westholm et $\mathrm{al}^{26}$. revealed that circRNAs 
substantially increase during CNS aging and that circRNAs represent a class of aging biomarkers in Drosophila. Liu et $\mathrm{al}^{27}$. identified a new circRNA involved in cartilage injury and OA development and progression, which suggests that circRNA-CER could be used as a potential target in OA therapy. The NP and cartilage share a common developmental lineage at the molecular level, which is the main functional component in IVD and consists of small chondrocyte-like cells with typical chondrocyte-like morphology ${ }^{19}$. To further document the involvement of circ-4099 in IVD degeneration, we detected the expression of circ-4099 in an IVDD cellular model created with TNF- $\alpha^{8}$. The results showed that TNF- $\alpha$ can induce the expression of circ-4099, which is consistent with previous data. Gain-of-function/loss-offunction studies were used to detect the exact role of circ4099 during NP cell matrix synthesis and degradation, which is crucial for normal disc function and results in spine mechanical stability. The results showed that circ4099 regulates the synthesis of Collagen II and Aggrecan, but not MMP3 and ADAMTS5. We also found that Sox9 is a target gene of circ- 4099 and that Sox 9 can positively regulate the expression of chondrocyte-specific genes, including aggrecan and collagen $\mathrm{II}^{22}$. In this way, circ4099 may achieve regulation of NP cells through Sox9.

Previous studies have demonstrated that the most activated cytokine pathways are the NF- $\mathrm{kB}$ and MAPK signaling pathways. To test whether a similar pattern is observed in the regulation of circRNAs in NP cells, we cotreated NP cells with NF- $\kappa B$ or MAPK pathway-specific inhibitors. We first used NF- $\kappa B$ pathway-specific inhibitors, which led to significant suppression of the induction of circ-4099 expression by TNF- $\alpha$ to almost the basal level. To detect the exact NF- $\kappa B$ pathway sub-unit involved in this regulation, we performed lentiviral p65/ IKK $\beta$ shRNA studies. The results showed that inhibition of TNF- $\alpha$-dependent circ-4099 expression was followed by suppression of p65 and IKK $\beta$ signaling, highlighting the importance of the NF- $\kappa B$ pathway in controlling circ4099 expression. Moreover, we observed that blocking the NF-kB pathway does not maintain the basal circ-4099 expression levels, which suggested that there might be a

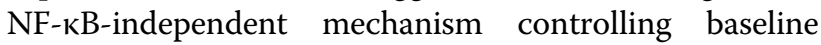
transcription. To further explore this regulatory system, we treated NP cells with MAPK pathway-specific inhibitors. The results showed that pretreatment with p38/JNK/ ERK inhibitors partially counteracted the inductive effects of TNF- $\alpha$, and there was differential sensitivity of circ4099 expression to MAPK isoforms. Based on these findings, it is reasonable to assume that cytokines regulate the expression of circ- 4099 by controlling the activity of specific MAPK isoforms and through NF- $\mathrm{KB}$ signaling in NP cells. Thus, the regulation of circ- 4099 by cytokines is more complex than was originally thought.
It has been reported that circRNAs can function as miRNA sponges; however, since most circRNAs show low abundance and have short lengths, many circRNAs may not serve as miRNA sponges ${ }^{12,28}$. In this study, we found that circ-4099 harbors miRNA-binding sites for miR-5085p, miR-561-5p, miR-616-5p, miR-328-5p, and miR-761. Although many miR target genes were predicted by miRNA software, only a few of them have been experimentally demonstrated to be direct targets in IVD degeneration. Sox9 is a miR-616-5p target gene related to IVD degeneration. Several studies have reported that miR-616 might play important roles in regulating cell proliferation, cell growth and migration, chondrogenic differentiation of human adipose-derived stem cells, and chondrogenesis $^{29,30}$. Previous studies have demonstrated that decreased Sox9 expression in NP cells is associated with IVD degeneration ${ }^{31}$. Sox 9 is a master regulator of NP cell differentiation, and Sox9 over-expression can enhance the expression of typical matrix protein-coding genes (Col2, Aggrecan, Versican, etc.) ${ }^{32,33}$. Sox9 over-expression can also reverse the upregulation of MMP-3, MMP-13, ADAMTS- 5 induced by IL- $1 \beta^{34}$, suggesting that Sox 9 could be a potential therapeutic target. The pivotal role of Sox9 in ECM degradation has been reported to be regulated by microRNAs, such as MicroRNA-30a, which promotes ECM degradation in articular cartilage via downregulation of Sox $9^{35}$; miR-101, which promotes condylar cartilage cell apoptosis by inhibiting Sox $9^{36}$; and MicroRNA-145, which regulates MSC chondrogenic differentiation by targeting $\operatorname{Sox} 9^{37}$. As the circRNA mechanism is complicated and many other molecules participate in the IVD degradation process, in this study, we only focused on miR-616-5p, which targets both circ4099 and Sox9. To verify this speculation, the binding effects of circ-4099 and miR-616-5p were demonstrated by FISH, RIP for AGO2, a RNA-RNA pull-down-qPCR assay and a luciferase reporter gene assay. Gain-offunction and loss-of-function studies were performed to demonstrate that circ-4099-regulated Sox9 expression.

Using DNA pull-down and spectrometry experiments, we found that TNF- $\alpha$ can promote circ-4099 transcription through GRP78 upregulation. GRP78 is known to be expressed in early embryonic development ${ }^{38}$ and protects against cell death by increasing the protein-folding capacity in the ER or redistributes to mitochondria to form complexes with $\mathrm{BIK}^{39}$. Our previous study indicated that the increased GRP78 in degenerative discs can be considered to be a negative-feedback mechanism to reset the stress sensors in the ER, which leads to increased ER protein-folding capacity, maintenance of disc cell integrity and survival ${ }^{4}$. The exact role and dedicated mechanism require further analysis.

In our study, we constructed a network that included circRNAs and mRNAs: a circRNA-miRNA-mRNA 
network. The network indicated the potential association between circRNAs and their target genes, and provided an important reference for studying the interactions between other differentially expressed circRNAs and their potential targets.

Collectively, this is the first systematic analysis of circRNA expression and its roles in IVD NP tissues. Our data indicate that 72 circRNAs were either over-expressed or under-expressed by more than twofold in IVDD, with Circ-4099 increased by approximately sixfold, which may have biological consequences on the progression of IVD degeneration. Over-expression of circ-4099 increased the expression of collagen II and Aggrecan. TNF- $\alpha$ promoted Circ-4099 expression through the MAPK and NF- $\mathrm{kB}$ pathways. We further confirmed that circ-4099 functions as a "sponge" by competitively binding miR-616-5p, which reverses the suppression of Sox 9 by miR-616-5p, and participates in ECM synthesis in NP cells.

A previous study showed that increased Sox9 can positively regulate the expression of chondrocyte-specific genes, including aggrecan and collagen $\mathrm{II}^{22}$. These results indicated that circ-4099 may be a protective gene. Thus, controlling circ-4099 activities during disc disease may restore NP cell functions and delay or reverse the IVDD process. Further studies should focus on the exact molecular mechanisms and pathophysiological roles of circ4099 in IVDD as a potential new therapeutic strategy for IVDD treatment.

\section{Acknowledgements}

This work was supported by grants from the National Natural Science Foundation of China (Grant No. 81401840 and 81371984), Sun Yat-sen University Starting Funds for Young Teachers (Grant No.16ykpy31), and the Guangdong Province Medical research fund project (No. 2016A030313259)

\section{Author details}

'Department of Orthopedics, First Affiliated Hospital of Sun Yat-sen University, 58 Zhongshan Second Road, Guangzhou, China. ${ }^{2}$ Department of Medical Genetics, Zhongshan School of Medicine and Center for Genome Research, Sun Yat-Sen University, 74 Zhongshan Second Road, Guangzhou, China

\section{Author contributions}

W.H. contributed to the study design and microarray analysis, identified specific circRNAs, and wrote the manuscript. H.P.H. and W.J.R. contributed to the cell regulation experiments and the RNA FISH analysis. P.H.H. collected the clinical samples and performed the RNA-protein interaction analysis. L.J., L.Z. M., and L.H. performed the in vitro analysis experiments.

J.W.Y. and Z.Z.M. contributed to the study design, collected the data and performed the data analysis. All the authors read and approved the final manuscript.

\section{Conflict of interest}

The authors declare that they have no conflict of interest.

\section{Publisher's note}

Springer Nature remains neutral with regard to jurisdictional claims in published maps and institutional affiliations.
Supplementary information accompanies this paper at https://doi.org/ 10.1038/s12276-018-0056-7.

Received: 26 September 2017 Accepted: 25 December 2017. Published online: 13 April 2018

\section{References}

1. van Dieen, J. H., Kuijer, P. P., Burdorf, A., Marras, W. S. \& Adams, M. A. Nonspecific low back pain. Lancet 379, 1874 (2012).

2. Wang, X. et al. Tumor necrosis factor-alpha- and interleukin-1 beta-dependent matrix metalloproteinase-3 expression in nucleus pulposus cells requires cooperative signaling via syndecan 4 and mitogen-activated protein kinaseNF-kappaB axis: implications in inflammatory disc disease. Am. J. Pathol. 184 2560-2572 (2014).

3. Livshits, G. et al. Lumbar disc degeneration and genetic factors are the main risk factors for low back pain in women: the UK Twin Spine Study. Ann. Rheum. Dis. 70, 1740-1745 (2011).

4. Wang, H. et al. Role of death receptor, mitochondrial and endoplasmic reticulum pathways in different stages of degenerative human lumbar disc. Apoptosis 16, 990-1003 (2011).

5. Wang, S. Z., Rui, Y. F., Lu, J. \& Wang, C. Cell and molecular biology of intervertebral disc degeneration: current understanding and implications for potential therapeutic strategies. Cell Prolif. 47, 381-390 (2014).

6. Roberts, S., Evans, H., Trivedi, J. \& Menage, J. Histology and pathology of the human intervertebral disc. J. Bone Jt. Surg. Am. 88(Suppl 2), 10-14 (2006).

7. Sun, F., Qu, J. N. \& Zhang, Y. G. Animal models of disc degeneration and major genetic strategies. Pain Physician 16, E267-E275 (2013).

8. Wang, H. et al. Inflammatory cytokines induce NOTCH signaling in nucleus pulposus cells: implications in intervertebral disc degeneration. J. Biol. Chem. 288, 16761-16774 (2013).

9. Jeck, W. R. et al. Circular RNAs are abundant, conserved, and associated with ALU repeats. RNA 19, 141-157 (2013).

10. Ashwal-Fluss, R. et al. circRNA biogenesis competes with pre-mRNA splicing. Mol. Cell 56, 55-66 (2014).

11. Li, F. et al. Circular RNA ITCH has inhibitory effect on ESCC by suppressing the Wnt/beta-catenin pathway. Oncotarget 6, 6001-6013 (2015).

12. Hansen, T. B. et al. Natural RNA circles function as efficient microRNA sponges. Nature 495, 384-388 (2013).

13. Hansen, T. B. et al. miRNA-dependent gene silencing involving Ago2mediated cleavage of a circular antisense RNA. EMBO J. 30, 4414-4422 (2011).

14. Ghosal, S., Das, S., Sen, R., Basak, P. \& Chakrabarti, J. Circ2Traits: a comprehensive database for circular RNA potentially associated with disease and traits. Front. Genet. 4, 283 (2013).

15. Salzman, J., Chen, R. E., Olsen, M. N., Wang, P. L. \& Brown, P. O. Cell-type specific features of circular RNA expression. PLoS Genet. 9, e1003777 (2013).

16. Zhang, X. O. et al. Complementary sequence-mediated exon circularization. Cell 159, 134-147 (2014)

17. Pfirmann, C. W., Metzdorf, A., Zanetti, M., Hodler, J. \& Boos, N. Magnetic resonance classification of lumbar intervertebral disc degeneration. Spine $\mathbf{2 6}$ 1873-1878 (2001).

18. Risbud, M. V. et al. Nucleus pulposus cells express HIF-1 alpha under normoxic culture conditions: a metabolic adaptation to the intervertebral disc microenvironment. J. Cell Biochem. 98, 152-159 (2006).

19. Hunter, C. J., Matyas, J. R. \& Duncan, N. A. Cytomorphology of notochordal and chondrocytic cells from the nucleus pulposus: a species comparison. J. Anat. 205, 357-362 (2004).

20. Enright, A. J. et al. MicroRNA targets in Drosophila. Genome Biol. 5, R1 (2003).

21. Pasquinelli, A. E. MicroRNAs and their targets: recognition, regulation and an emerging reciprocal relationship. Nat. Rev. Genet 13, 271-282 (2012).

22. Li, P. et al. Estrogen enhances matrix synthesis in nucleus pulposus cell through the estrogen receptor beta-p38 MAPK pathway. Cell Physiol. Biochem. 39, 2216-2226 (2016).

23. Lewis, B. P., Burge, C. B. \& Bartel, D. P. Conserved seed pairing, often flanked by adenosines, indicates that thousands of human genes are microRNA targets. Cell 120, 15-20 (2005).

24. Marat, A. L., Dokainish, H. \& MCPherson, P. S. DENN domain proteins: regulators of Rab GTPases. J. Biol. Chem. 286, 13791-13800 (2011).

25. Li, Y. et al. Cancer driver candidate genes AVL9, DENND5A and NUPL1 contribute to MDCK cystogenesis. Oncoscience 1, 854-865 (2014). 
26. Westholm, J. O. et al. Genome-wide analysis of drosophila circular RNAs reveals their structural and sequence properties and age-dependent neural accumulation. Cell Rep. 9, 1966-1980 (2014).

27. Liu, Q. et al. Circular RNA related to the chondrocyte ECM regulates MMP13 expression by functioning as a MiR-136 'Sponge' in human cartilage degradation. Sci. Rep. 6, 22572 (2016).

28. Rybak-Wolf, A. et al. Circular RNAs in the mammalian brain are highly abundant, conserved, and dynamically expressed. Mol. Cell 58, 870-885 (2015).

29. Zhang, D. et al. MicroRNA-616 promotes the migration, invasion and epithelial-mesenchymal transition of HCC by targeting PTEN. Oncol. Rep. $\mathbf{3 5}$ 366-374 (2016)

30. Ma, S. et al. MicroRNA-616 induces androgen-independent growth of prostate cancer cells by suppressing expression of tissue factor pathway inhibitor TFPI2. Cancer Res. 71, 583-592 (2011).

31. Gruber, H. E., Norton, H. J., Ingram, J. A. \& Hanley, E. N. Jr. The SOX9 transcription factor in the human disc: decreased immunolocalization with age and disc degeneration. Spine 30, 625-630 (2005).

32. Lee, Y. J., Kong, M. H., Song, K. Y., Lee, K. H. \& Heo, S. H. The relation between Sox9, TGF-beta1, and proteoglycan in human intervertebral disc cells. J. Korean Neurosurg. Soc. 43, 149-154 (2008).
33. Sun, W. et al. Sox9 gene transfer enhanced regenerative effect of bone marrow mesenchymal stem cells on the degenerated intervertebral disc in a rabbit model. PLOS ONE 9, e93570 (2014).

34. Lu, $\mathrm{H}$. et al. Lentiviral vector-mediated over-expression of Sox9 protected chondrocytes from IL-1beta induced degeneration and apoptosis. Int J. Clin. Exp. Pathol. 8, 10038-10049 (2015)

35. Chang, T. et al. MicroRNA-30a promotes extracellular matrix degradation in articular cartilage via downregulation of Sox9. Cell Prolif. 49, 207-218 (2016).

36. Li, X., Wang, Z. X., Wang, Z. S. \& Li, Q. F. Effect of microRNA-101 on apoptosis of rabbit condylar cartilage cells by inhibiting target gene SOX9. Asian Pac. J. Trop. Med 8, 502-505 (2015).

37. Yang, B. et al. MicroRNA-145 regulates chondrogenic differentiation of mesenchymal stem cells by targeting Sox9. PLOS ONE 6, e21679 (2011).

38. Sun, F. C. et al. Localization of GRP78 to mitochondria under the unfolded protein response. Biochem J. 396, 31-39 (2006).

39. Zhang, Y. H., Zhao, C. Q., Jiang, L. S. \& Dai, L. Y. Cyclic stretch-induced apoptosis in rat annulus fibrosus cells is mediated in part by endoplasmic reticulum stress through nitric oxide production. Eur. Spine J. 20, 1233-1243 (2011). 